\title{
Ni-Mn-Ga Single Crystal Exhibiting Multiple Magnetic Shape Memory Effects
}

\author{
Oleg Heczko $^{1} \cdot$ Petr Veřtát $^{1} \cdot$ Marek Vronka $^{1,2} \cdot$ Vít Kopecky $^{1} \cdot$ Oleksiy Perevertov $^{1}$
}

Published online: 22 September 2016

(c) ASM International 2016

\begin{abstract}
Both magnetically induced phase transformation and magnetically induced reorientation (MIR) effects were observed in one $\mathrm{Ni}_{50} \mathrm{Mn}_{28} \mathrm{Ga}_{22}$ single crystal sample by direct measurement of the magnetic field-induced strain. We investigated various twinning microstructures ranged from single twin interface to fine twinning and crossing twins to evaluate what controls the apparent twinning stress crucial for MIR. The main challenges for the applications of these effects are outlined.
\end{abstract}

Keywords Magnetic shape memory .

NiMnGa $<$ materials · Stress-strain · Twinning · Magnetic field-induced transformation - Magnetic field-induced reorientation

\section{Introduction}

The magnetic shape memory (MSM) alloys are the class of materials exhibiting multiferroic behaviour combining ferroelasticity and (ferro)magnetism [1]. The magnetic shape memory effect is a general name for several effects, in which the most visible feature is huge strain induced by magnetic field $[2,3]$. It is similar to conventional shape memory effect but the shape of the material is controlled by magnetic field. In comparison with thermal

Oleg Heczko

heczko@fzu.cz

1 Institute of Physics, Academy of Science of the Czech Republic, Na Slovance 2, 18221 Prague, Czech Republic

2 Department of Materials, Faculty of Nuclear Sciences and Physical Engineering, Czech Technical University in Prague, Trojanova 13, 12000 Prague, Czech Republic (conventional) shape memory, the force output is not large but it is much faster and in comparison with electrostriction it can be achieved on distance. Outstanding example exhibiting all various effects is $\mathrm{Ni}-\mathrm{Mn}-\mathrm{Ga}$ low symmetry phase. This phase called martensite is a product of martensitic transformation of cubic, $\mathrm{L} 2{ }_{1}$ ordered, close to stoichiometric $\mathrm{Ni}_{2} \mathrm{MnGa}$ Heusler phase.

To date the most investigated phenomenon is magnetic field-induced strain called magnetic field-induced structure reorientation (MIR). The effect takes place in single twinned phase called martensite by magnetic field-induced motion of twin boundaries. The twin variant favourably oriented in magnetic field grows at the expenses of the other variants which results in the reorientation of the microstructure [4-6]. The maximum strain is then given by the difference between lattice constants of martensite $\varepsilon_{\mathrm{MIR}}=1-a_{\mathrm{M}} / c_{\mathrm{M}}$. The driving force is determined by the difference of the magnetic energy $\Delta E_{\text {mag }}$ between martensitic variants with different orientation of the magnetization [2, 6]. In the $\mathrm{Ni}-\mathrm{Mn}-\mathrm{Ga}$ martensite, it results in giant deformations of up to $12 \%$ in moderate magnetic fields less than $1 \mathrm{~T}$ [7].

Importantly, no phase transformation is involved in this case in contrast to another effect which can be called magnetic field-induced phase transformation [8]. Which phase is induced by magnetic field depends on the difference between saturation magnetizations of the phases. Consequently, it is called magnetically induced austenite (MIA) if saturation magnetization of austenite is larger or magnetically induced martensite (MIM) if saturation magnetization of martensite is larger. It can be viewed as the stabilization of the phase with higher magnetization or as the shift of transformation temperature due to magnetic field. This provides the full analogy to the stress-induced transformation in conventional shape memory alloys. The 
field-induced martensitic transformation can give rise to large strain due to difference of lattice constants of austenite and martensite, i.e. $\varepsilon_{\mathrm{MIM}}=1-a_{\mathrm{A}} / c_{\mathrm{M}}$ or $1-$ $a_{\mathrm{A}} / a_{\mathrm{M}}$. The strength of magnetic field needed for MIM/ MIA is of the order of magnitude larger than for the reorientation (MIR). Figure 1 shows the field-induced strain mechanism of both effects to emphasize the difference between them.

Apart of the strain mechanism, another crucial difference between MIR and MIM/MIA is the energy output provided by magnetic field [2]. While in MIR the energy for reorientation and thus work output is intrinsically limited by magnetocrystalline anisotropy and saturation magnetization of the martensite, for MIM in principle the energy is unlimited as it is given by $\Delta \mathrm{MH}$, where $\mathrm{H}$ is magnetic field and $\Delta M=M_{\mathrm{s}}^{\mathrm{M}}-M_{\mathrm{s}}^{\mathrm{A}}$ is the difference of saturation magnetization of both phases and $M_{\mathrm{s}}^{\mathrm{M}}>M_{\mathrm{s}}^{\mathrm{A}}$. This is valid for large magnetic field securing the magnetic saturation of both phases. In contrast, for MIR the increase of magnetic field above anisotropy field has no effect [9]. This simple concept of MIR mechanism [4, 6, 9] is, however, disputed and there is alternative explanation based on magnetoelasticity [10-12] which suggests that MIR can occur in the phase with low magnetocrystalline anisotropy.

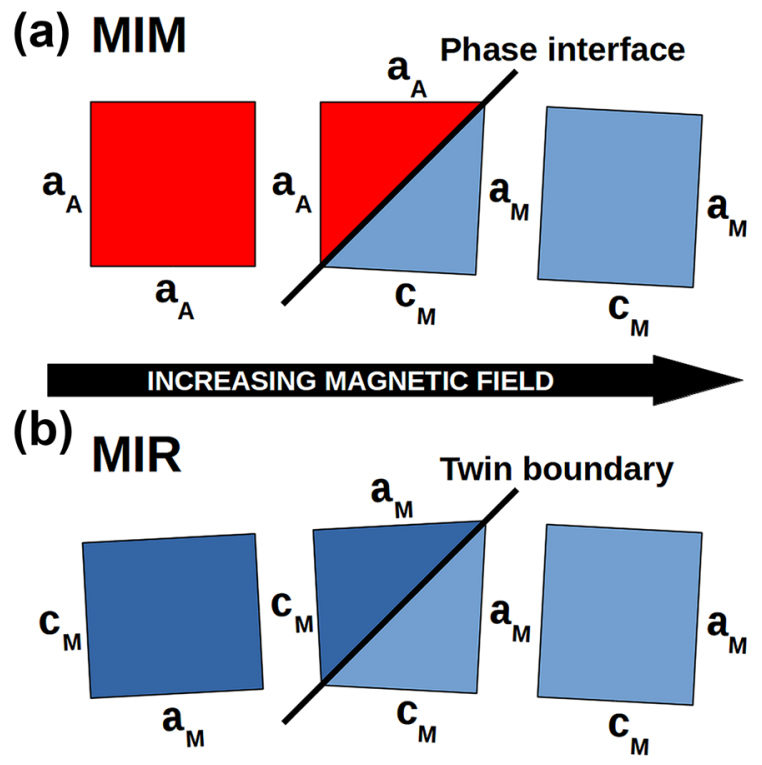

Fig. 1 Schematic 2D drawing of the mechanism of magnetic fieldinduced effects in ferromagnetic and ferroelastic Ni-Mn-Ga showing the difference between magnetically induced phase transition to tetragonal martensite (MIM) and magnetically induced reorientation (MIR) between two tetragonal twin variants. The maximum strain is determined by lattice constants of cubic austenite, $a_{\mathrm{A}}$, and tetragonal martensite $a_{\mathrm{M}}$ and $c_{\mathrm{M}}$. For the sake of clarity, the difference (10\%) of lattice constants is exaggerated. The inclination of the unit cell corresponds to lattice rotation between austenite and martensite and between two tetragonal variants of martensite. Red austenite, blue martensite, shades mark the different orientation (Color figure online)
Contrary to many observations dealing with material with high anisotropy, there is no direct experimental proof. The microscopic mechanism is discussed in [13].

In summary, the principal demands of the magnetic properties for the effects are: the magnetic anisotropy and twin boundary mobility as large as possible for MIR; the difference of saturation magnetization as large as possible for MIM, ultimately having material with one phase that is not magnetic, i.e. $M_{\mathrm{s}}$ is zero. Also small thermal hysteresis is important to secure reversibility of MIM, which is a crucial demand for magnetocaloric applications.

The described effects in magnetic field have a direct analogues to conventional shape memory, i.e. stress-induced reorientation or pseudoplasticity, stress-induced transformation or superelasticity, and shape memory behaviour. All these effects were also demonstrated in $\mathrm{Ni}-$ Mn-Ga. However, the multiferroic character of the material gives another possibility to obtain interesting and applicable effects. By combining magnetic field and mechanical force we can obtain reversible MIR and reciprocal effect of magnetically induced superelasticity [2, $13,14]$ or or the stress-assisted MIM and reverse effect of magnetic field-assisted mechanical superelasticity. Closely associated to MIM is an effect called (magnetic) stray fieldinduced microstructure (SFIM) which is in principle thermally induced transformation followed by spontaneous detwinning of thermally induced martensite microstructure due to demagnetization [15]. This effect thus can occur only in flat sample or rather thin film. Another promising field is vibration damping, particularly the magnetic fieldcontrolled damping [16] and energy harvesting [17]. All these multiple functionalities are based on various combinations of magnetic field, mechanical stress and temperature.

Furthermore, manipulating the extrinsic magnetic properties of material we can obtain additional functionality, e.g. by increasing magnetic hysteresis, we can obtain permanent magnets that can be mechanically demagnetized $[18,19]$. However, for all the promises and exciting possibilities one should be aware of the limits. While for MIM there is no additional material demand apart from the conditions necessary for the effect, the significant MIR can be expected only in single crystals or strongly textured polycrystals (oligocrystals) [20] as grain boundaries form insurmountable obstacles for twin boundary motion.

To date the largest challenge is to improve the materials' properties such as the relatively low temperature of martensitic transformation which limits the applicability of the effects [7, 9]. For MIR, another challenge is to secure high mobility of twin boundaries in repeatable fashion and in general to prepare favourable twinned microstructure, which is a task for domain engineering [21, 22]. It is a necessary condition for applications such as actuation, 
damping, and energy harvesting. Moreover, there is only limited knowledge about fatigue behaviour. Several million cycles of repeatable twin boundary motion in the single phase were demonstrated [23, 24]; however, the material integrity is much more difficult to secure in the case of phase transformation, i.e. for MIM.

Although both effects, i.e. MIR and MIM, are known for some time and they were separately observed in various materials, here for the first time, we experimentally demonstrate both effects on one sample revealing multiple functionalities of the Ni-Mn-Ga single crystal. Additionally, we describe some twin attributes of the single crystal $\mathrm{Ni}-\mathrm{Mn}-\mathrm{Ga}$ necessary for magnetically induced reorientation (MIR) and provide a comparison between single crystals from previous and recently improved metallurgy production to search for the conditions of the extreme high mobility of twin boundaries.

\section{Experimental Methods}

For investigation of different magneto-elastic effects, we used single crystal with size $10 \times 2 \times 1$ millimetres and composition $\mathrm{Ni}_{50} \mathrm{Mn}_{28} \mathrm{Ga}_{22}$ prepared by modified Bridgman method in Adaptamat Ltd. For comparison we used also sample prepared at HUT in the year 2000 with size $10 \times 5 \times 5$ millimetres. At room temperature the crystals were in martensitic state with modulated $10 \mathrm{M}$ structure which can be approximate by monoclinic lattice. Typical lattice parameters were $a=0.596 \mathrm{~nm}, b=0.594 \mathrm{~nm}$, $c=0.560 \mathrm{~nm}, \gamma=90.3^{\circ}$ using the lattice unit cell and orientation inherited from cubic austenite. The transformation temperature to austenite was about $320 \mathrm{~K}$. All samples were parallelepipeds with faces approximately $\{100\}$ planes of austenite.

For twin variants identification, we used optical microscopy and SEM. The twins can be identified by surface relief using Nomarski contrast and from optical activity of martensite using polarization contrast. Each method is sensitive to different twin orientations. The identification from surface relief might be difficult due to polishing artefacts, i.e. the previous twinned structure can be polished out flat but it springs up back during transformation or reorientation-one can call it the spirits of dead martensite, phantom twins or historical twins [22]. To recognize this feature one has to check the surface in austenite in which the surface should be flat and featureless. Observation in polarization light removes this difficulty of separating the artefacts from real twinning; however, only twin orientations optically active can be observed.

Stress-strain curves due to reorientation were measured by custom-made miniaturized stress-strain machine with maximum force of $10 \mathrm{~N}$ (as very small twinning stress is involved) and for large samples INSTRON 1362 electromechanical testing machine was used. Magnetization measurements indicating the twin boundary movement in magnetic field were done using vibrating sample magnetometer PAR at room temperature.

Dilatation in magnetic field was measured inside superconducting magnet Cryogenic using capacitance sensor consisting of two parallel copper annuli as plates. The sample was fixed between these plates without additional fixture. The relation between distance of the plates and capacitance was experimentally calibrated with good agreement with theoretical value for flat capacitor. Ultraprecise AndeenHagerling AH 2550A Capacitance Bridge (resolution 0.8 aF) was used for capacitance measurement allowing to measure dilatation with the resolution $0.1 \mathrm{ppm}$. To measure the dilatation, the sample was compressed with small force. The directions of force, magnetic field and measured strain were collinear as shown in the inset of Fig. 2.

\section{Results}

As the martensitic transformation is (approximately) volume conserving transformation, the transformation to low symmetry phase can result in large strain. The sharp strain change measured upon thermoelastic transformation is shown in Fig. 2. The sharp transformation reveals good

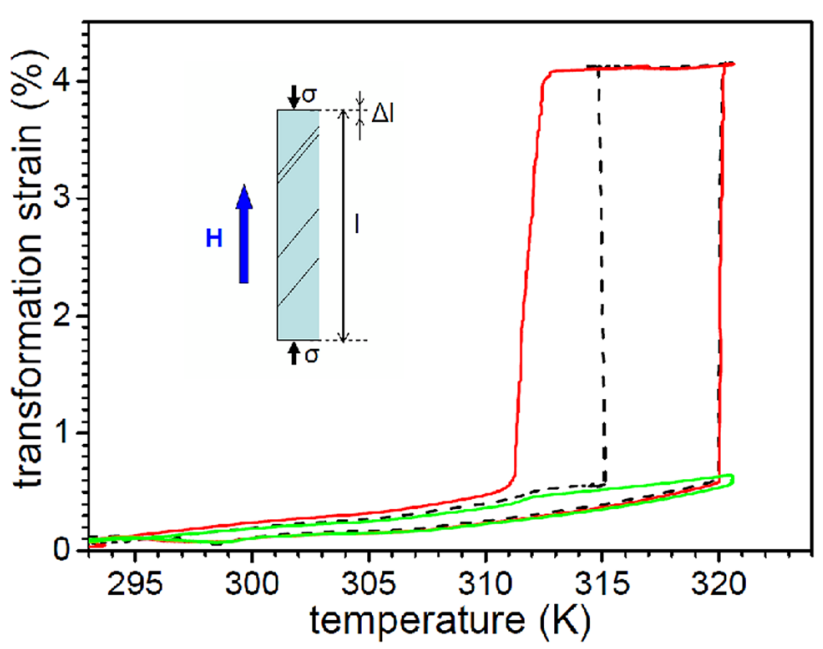

Fig. 2 Transformation strain during field-assisted thermally induced martensitic transition (three different thermal cycles marked by different colour lines). Red: in zero magnetic field. Black broken line: after application of magnetic field $5 \mathrm{~T}$ in austenite during cooling resulting in the shift of reverse transformation temperature to higher value. Green: after application of magnetic field $5 \mathrm{~T}$ at room temperature in martensite during heating, it shifted the transformation temperature above measuring range (the sample remained in martensite state). The inset shows schematically the measuring geometry. Twin boundaries or phase interfaces are symbolically marked by few diagonal lines (Color figure online) 
quality of single crystal without significant chemical inhomogeneity. To obtain largest strain the sample was compressed before measurement to prepare single variant state with c-axis along stress and in the direction of magnetic field and strain measurement. In martensite measured thermal expansion was not linear and relatively large. This agrees with the large and nonlinear change of $c$ lattice constant with temperature in the vicinity of martensitic transformation measured by X-ray diffraction [25]. Without magnetic field, the transformation to cubic austenite occurred at $320.5 \mathrm{~K}$ and reverse transformation to martensite started at $312.5 \mathrm{~K}$. The sample transformed back to approximately single variant due to stress $\sim 1 \mathrm{MPa}$ applied to upper moving plate in dilatometer device to fix the sample. If no back-stress were applied the sample might transform spontaneously to multivariant state and the thermal strain loop may not be closed or even the strain could be zero or negative [26]. However, recently Pinneker et al. [27] demonstrated reversible large strain change upon heating in thin foils. Large reversible deformation may be due to high demagnetization factor of thin foil preferring c-axis in-plane. This is then analogous to the observed SFIM effect in thin films [15].

By applying constant magnetic field $5 \mathrm{~T}$ in austenite during cooling the martensite phase was preferred $\left(\mathrm{M}_{\mathrm{s}}{ }^{\mathrm{M}}>\right.$ $\mathrm{M}_{\mathrm{s}}^{\mathrm{A}}$ ) and the transformation temperature to martensite was shifted to higher temperature about $315 \mathrm{~K}$. If the field was applied in martensite at room temperature during heating, the transformation to austenite was also shifted to higher temperature due to martensite stabilization. Unfortunately, due to limitation of the measuring device we could not go beyond $321.5 \mathrm{~K}$ and no transformation was detected. The observed temperature shift is relatively small due to small difference of magnetization of austenite and martensite which is caused by different Curie point of the phases. The temperature shift of $0.5 \mathrm{~K} / \mathrm{T}$ is in agreement with previous measurements $[2,3]$ and theoretical estimation from the Maxwell relations [3].

At constant temperature, above but in close vicinity of the transformation, the transformation to martensite from austenite can be induced by increasing magnetic field (MIM). Figure 3 shows the evolution of the deformation with increasing magnetic field at $314 \mathrm{~K}$. The initial increase of the field results only in very small magnetostriction strain and at about $4 \mathrm{~T}$ the transformation from austenite to martensite occurred. It proceeds by nucleation and movement of phase boundaries as marked in Fig. 1. As the transformation occurred at high field, we can reasonably expect that the materials transformed to single variant with easy magnetization axis, i.e. with c-axis along the field. The maximum deformation is then determined by the difference of lattice constants of the phases $\varepsilon=1-a_{\mathrm{A}} / c_{\mathrm{M}}$ (Fig. 1). The transformation is relatively sharp that can be

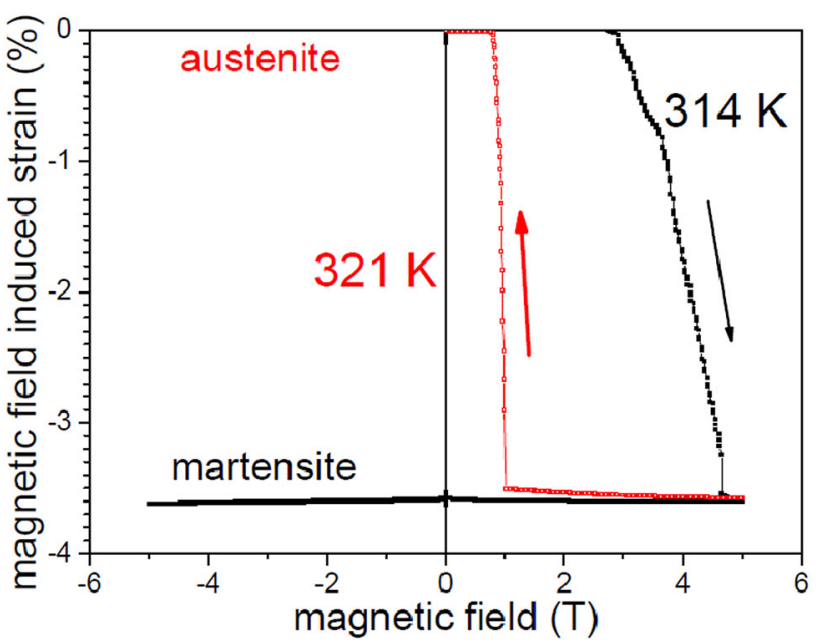

Fig. 3 Strain during magnetic field-induced transformation (MIM) at constant temperature. At increasing field at $314 \mathrm{~K}$ the sample in austenite transformed to martensite. The sample stayed in martensite after switching off the field. To obtain the reverse transformation from martensite to austenite, the sample was heated in $5 \mathrm{~T}$ to $321 \mathrm{~K}$, i.e. above the temperature of zero-field transformation. The decrease of the field resulted in the reappearance of austenite as the martensite is not any more stabilized by the field. The geometry of measurement is shown in the inset of Fig. 2

ascribed to single crystallinity and homogeneity of the sample. There is no significant change of the strain (in this resolution) with decreasing magnetic field. The sample remains in martensite due to thermal hysteresis of the transformation. With the hysteresis about $8 \mathrm{~K}$ and the shift $0.5 \mathrm{~K} / \mathrm{T}$ the full reversibility at constant temperature can be achieved only at large magnetic field above $16 \mathrm{~T}$ and at temperature more than $8 \mathrm{~K}$ above zero-field transition temperature. Here, we can achieve the reverse transformation to austenite with decreasing field only by increasing the temperature as demonstrated in Fig. 3. The sample was heated to $321 \mathrm{~K}$ and kept constant. Then the magnetic field was decreased and the field-stabilized martensite transformed back to austenite at about $1 \mathrm{~T}$ in agreement with the observed temperature shift of $0.5 \mathrm{~K} / \mathrm{T}$.

Full reversibility is much easier to achieve in similar Heusler alloy as Ni-Mn-In-Co with large difference of saturation magnetization. In such alloy, the martensite magnetization is close to zero and the transformation temperature exhibits a shift of more than $5 \mathrm{~K} / \mathrm{T}$. Thus, relatively small field of $1.5 \mathrm{~T}$ is enough to induce transformation. This was demonstrated for bulk alloys [28] and importantly also for thin films [29]. The reversible transformation is often, but not quite precisely, called metamagnetic behaviour and it is expected to be important for magnetocaloric applications [3, 30].

To obtain magnetically induced reorientation (MIR) the sample must contain the highly mobile twin boundaries between martensitic variants with different crystal 
orientations. By tensile stress, we prepared a single variant sample with a-axis along the field, i.e. with $\mathrm{c}$-axis perpendicular to the field. In this case, the reorientation from the variant with a-axis along the field to the variant with c-axis along the field provides the maximum strain (Fig. 1b). However, to measure the dilatation, the sample was fixed and compressed resulting in partial mechanical reorientation prior to the application of magnetic field. This mechanical reorientation occurred thanks to easy nucleation of other variants and highly mobile twin boundaries [31]. Therefore, the measured strain was then lower. The results are shown in Fig. 4. Neglecting the magnitude of the strain, the basic features of MIR are well apparent. MIR occurred and becomes saturated in much smaller field than MIM. At first, there was large non-reversible part corresponding to the twin boundary sweeping across the sample. It saturated in larger field. After decreasing the field, a reversible part forming the butterfly pattern was apparent. Although the pattern superficially reminds the magnetostriction, it can be excluded based on the relatively large hysteresis of MIR curve in comparison with nearly hysteresis-free magnetization curve. The minor reversible deformation originated from particular twin boundaries configuration, in which twin boundaries repel each other [32]. This is supported by optical observation of residual twins of different orientations after measurement. This small reversible part of deformation is comparable to magnetostrictive strain measured for austenite (not shown). The observation also shows that we can obtain a reversible effect without any mechanical preload perpendicular to field, which is the usual configuration for reversible MIR $[2,9,33]$.

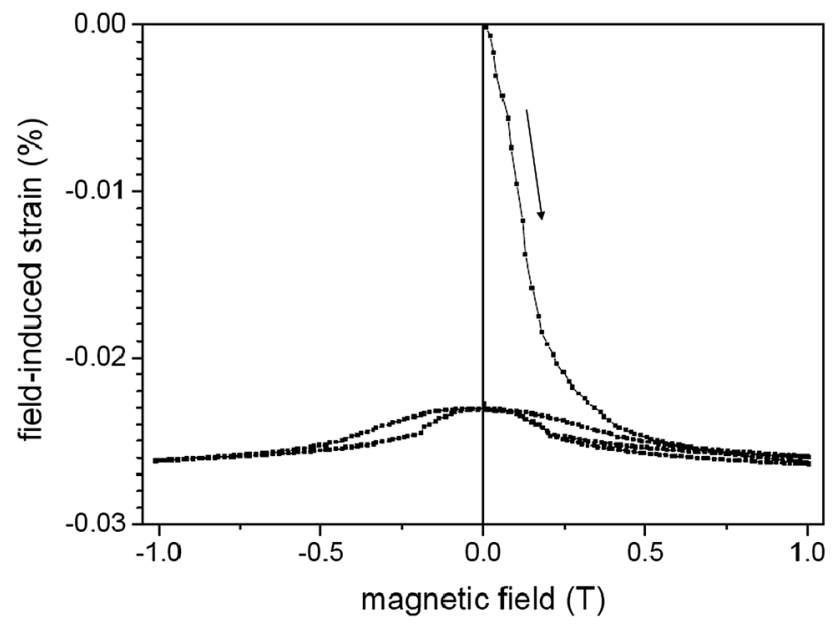

Fig. 4 The residual strain due to magnetically induced reorientation (MIR) after the application of magnetic field at room temperature. The magnitude of the strain is low due to compression induced by sample fixing along the field, i.e. the sample is nearly fully reoriented. The geometry of measurement is shown in the inset of Fig. 2
As the magnetic field is relatively weak force and, moreover, the magnitude of magnetic stress is limited, the highly mobile twin boundaries are crucial demands for the existence of MIR. The mobile boundary can be blocked in various ways, particularly by crossing twinning systems and the simplest configuration of twin boundaries is desirable. Figure 5 shows optical micrographs of the three simplest twin boundary configurations, single twin boundary of Type I, II and fine twinning of Type I. Two different types of mobile twin boundary arise due to slight monoclinicity of the martensite lattice [34]. This results in complex twin arrangement of various twinning systems $[35,36]$ which, however, does not decrease the mobility of twin boundaries by measurable amount.

All configurations (single boundary of Type I and Type II and multiple Type I boundary) were formed in the same sample and approximately the same place. This allows direct comparison between twin types and using the simple configurations the different mobility of Type I and II twin boundary can be accessed $[31,37]$. The micrographs show that the highly mobile twin boundary is a macroscopic entity that penetrates or crosses whole single crystal separating the two variants with different crystal orientations. Therefore, it is easy to assume that any imperfection inside the crystal [38] and on surface [14] can hinder its movement and thus the high perfection of the crystal is on demand. The mechanism of the twin boundary motion and origin of this "supermobility" in Ni-Mn-Ga is not quite known and further discussion is out of scope of the paper.

Figure 6 shows the stress-strain curves for different twin configurations. The plateau on the curve indicates the movement of twin boundary and it provides the definition of the stress needed for twin boundary motion called twining stress. This gives the exact description for weakly defined "mobility of twin boundary". The stress-strain curve exhibits plateau for single boundary while for fine boundaries configuration there is no plateau and stress is increasing nearly monotonously. The difference of twinning stress for Type I and II is ascribed to the different character of twin boundaries [36] but the origin is rather puzzling. The disappearance of plateau for fine twins is due to mutual interaction between the individual boundaries and possibly also due to magnetic interaction between differently oriented twins. The sharp but minuscule irregularly repeated decreases can be ascribed to the annihilation of individual twin boundaries.

To enlighten the importance of the crystal quality and proper twin structure, we compared these samples with the crystal from initial years of MSM research. The stressstrain curve and observed twin structure of this crystal is shown in Fig. 7. The twinning stress varies around 1.5 MPa during compression but it always remained above $1 \mathrm{MPa}$ as observed for single twin boundary. Larger 

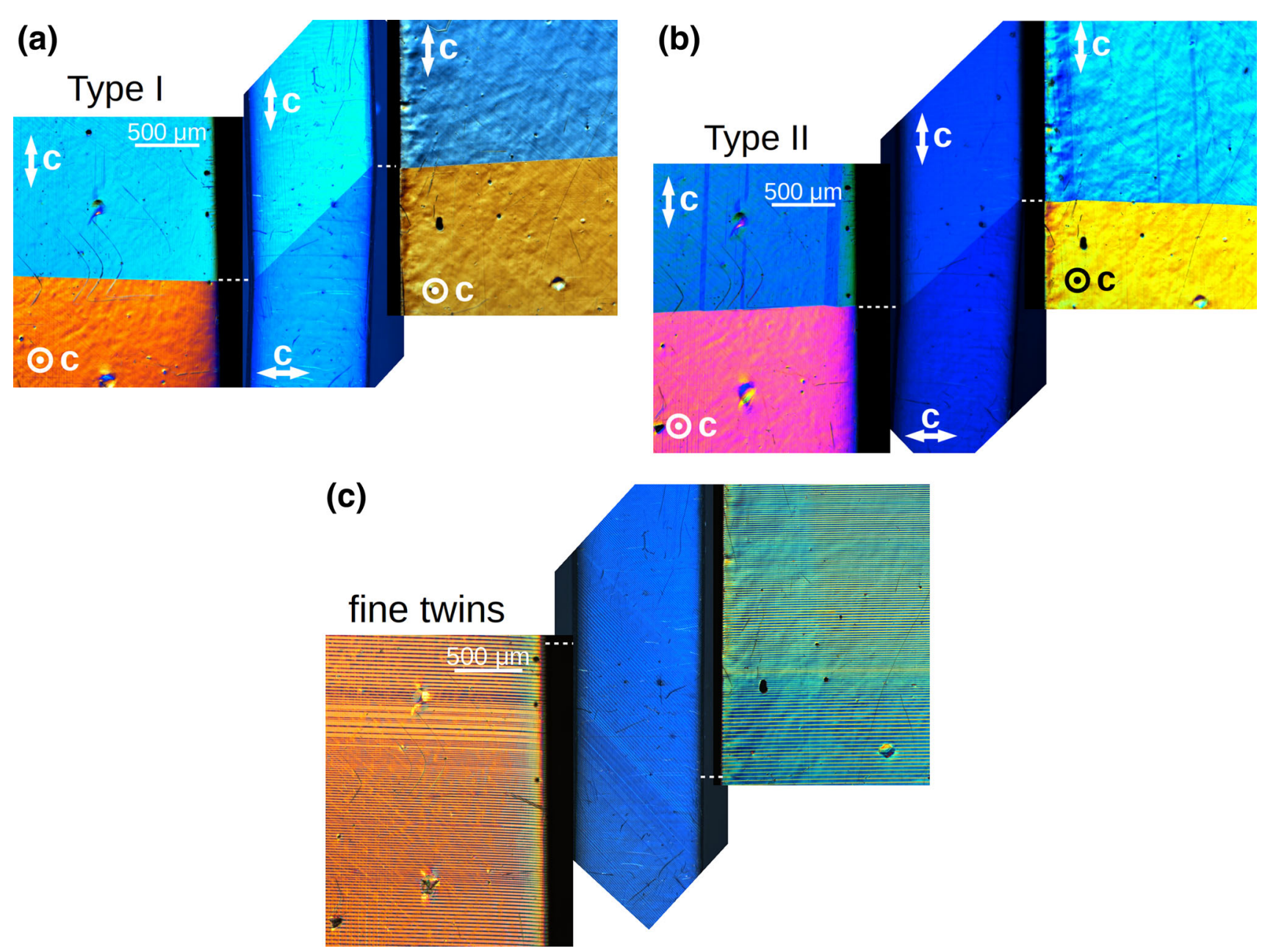

Fig. 5 Built-up optical micrographs of the projection of ferroelastic twin boundaries from the three sides of the sample. The $\mathrm{a} / \mathrm{c}$ twin boundary is approximately along (101) plane. The twin structure is formed mechanically; a single Type I, b single Type II, the boundary

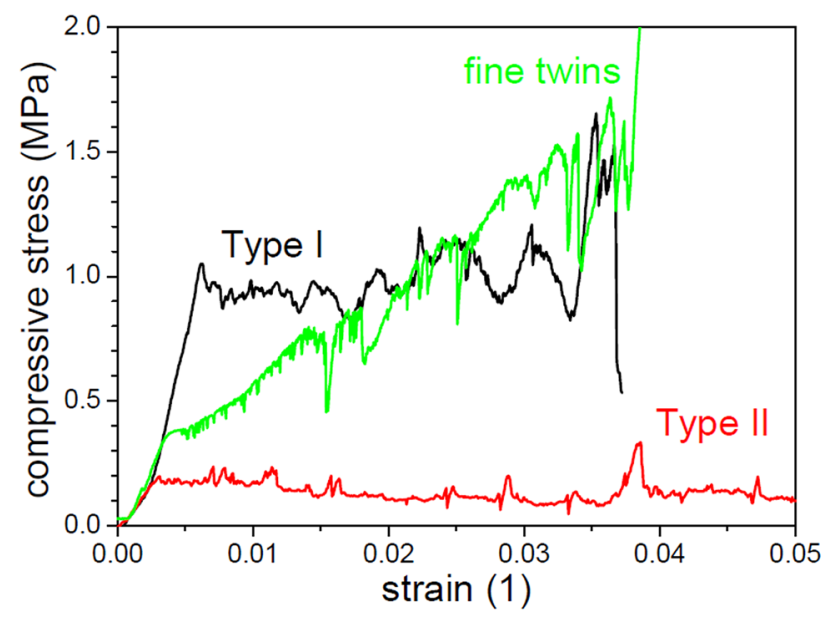

Fig. 6 Stress-strain curves of the twin arrangements shown in Fig. 5. The type of twinning is marked in the figure. Type I exhibited about $1 \mathrm{MPa}$ twinning stress; large drop at the end of stress-strain curve is caused by the additional formation of Type II boundary is slightly tilted and partly zigzagged due to modulation twinning ( $\mathrm{ew}$ vertical bands), $\mathbf{c}$ fine twins of Type I. The contrast in sides arises due to Nomarski contrast on the surface relief; the contrast in the middle is due to polarization contrast

twinning stress can be attributed partly to imperfections in the crystal and partly to impossibility to prepare well-ordered twin microstructure. In this crystal, the density of the voids (bubbles) and cracks was much higher than in new crystals analysed above. Moreover, although we started from the single variant, during compression apart from usual broad twin plates, we observed locally formed island of crossing twins (inset of Fig. 7). These twinning microstructures may further hinder the reorientation and increase the twinning stress. Such microstructures apparently form due to the presence of the crystal imperfections and cracks. Although these crossing twins increased the twinning stress significantly compared to the stress of single Type I boundary, they did not block the reorientation.

Surprisingly, the twinning stress did not change significantly for the sample after thermal transformation from austenite. In this case, spontaneous twinning microstructure is established in the sample and higher twinning stress is 


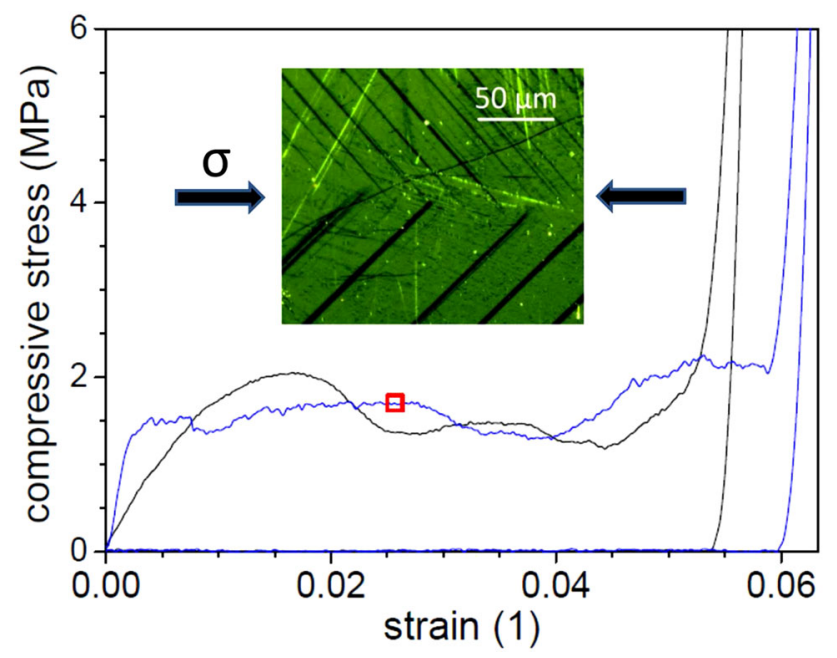

Fig. 7 Stress-strain curves of previously studied single crystal. Blue the curve of well-trained sample. The sample was initially compressed to single variant state and thus the maximum strain corresponds to full reorientation and it is given by lattice constants. No control can be exercised to form twin boundary during test. Optical micrograph in the inset shows, apart from broad twin bands, the crossing twins appearing in the sample during reorientation (position marked by hollow square). Stress-strain curve of the sample with spontaneously established twin microstructure after cooling from austenite (black). The maximum deformation is smaller due to initial state with multiple variants (Color figure online)

expected, particularly in the onset of the reorientation as observed previously [39]. The nearly negligible effect of transformation on twinning stress might be ascribed to many previous mechanical and magnetic field reorientation cycles which may constitute some kind of training of the twin microstructure [21, 22] and the memory of this training survive even in austenite. However, the basis of such training is not clear.

The twinning stress is a crucial parameter for MIR as it determines the magnitude of magnetic field in which MIR happens and determines the efficiency of the effect. There is a model which establishes the direct equivalence between mechanical stress and magnetic field $[2,6,9,40]$. The flat stress-strain plateau corresponds to sudden reorientation in magnetic field and measured switching field from magnetization curves agreed well with the measured twinning stress [41].

The extremely low twinning stress in modulated Ni$\mathrm{Mn}-\mathrm{Ga}$ single crystal is quite exceptional. There is a report about $1 \mathrm{MPa}$ twinning stress for compound twinning $\mathrm{Cu}-$ $\mathrm{Ni}-\mathrm{Al}$ single crystal [42]. However, for Type II twin boundary, the twinning stress was about $20 \mathrm{MPa}$ in compression and no Type I boundary has been identified to date. The structure of the martensite can be considered as an analogue of modulated structure in Ni-Mn-Ga [43].
Further research on $\mathrm{Cu}-\mathrm{Ni}-\mathrm{Al}$ single crystals is planned in search for non-magnetic analogue of Ni-Mn-Ga.

\section{Conclusion}

Both MIM and MIR effects were demonstrated in one $\mathrm{Ni}_{50} \mathrm{Mn}_{28} \mathrm{Ga}_{22}$ sample. It reveals multiple functionalities of this Heusler alloy. While for MIM there is no particular demand for martensitic structure, the conditions for MIR are much stricter. There must be particular structure of martensite and twinning microstructure with highly mobile twin boundaries or very low twinning stress. We demonstrated that the twinning microstructure controls the apparent twinning stress. Single interface of Type II exhibited the lowest twining stress.

While extreme low twining stress or high mobility is assumed to be connected with shear instability of the lattice, the origin of this "supermobility" in Ni-Mn-Ga is not quite known. Understanding this feature constitutes perhaps the main challenge in this field. There is no other material known to exhibit such low twining stress.

Concerning the applications, the major challenge is relatively low temperature of martensitic and also ferromagnetic transformations. It may be increased by substitution or doping by other elements in common Ni-Mn-Ga alloys [4, 44], but at the same time the particular martensite structure must be maintained. It is well demonstrated by low temperature limit. Off-stoichiometric $10 \mathrm{M}$ martensite transforms to other martensite phase at low temperature and MIR effect ceased [45].

As the exemplar shown here, another problem for the applications of MIR is the sensitivity of the effect on crystal quality and proper twin structure. Preparing suitable twin structure can be challenging in bulk but it seems nearly impossible for thin films. The domain engineering must solve how to form and modify twin boundary pattern for given purpose/applications (actuating, damping, shape morphing [46, 47], energy harvesting [17] and similar). This is particularly challenging for miniaturization. Up-to-date, there is no demonstration of MIR in free-standing films despite the initial promises [48]. On the other hand, there is no problem with MIM in films as it depends only on magnetization of the phases. Thus, the films exhibiting MIM can be suitable for (micro)magnetocaloric applications. Moreover, well-tailored films can withstand high number of transformation cycles as demonstrate for $\mathrm{Ni}-\mathrm{Ti}-\mathrm{Cu}[49]$.

Acknowledgments Authors thank L. Straka for discussion and hints improving the manuscripts. The work was supported by Czech Scientific Foundation Grant No. 14-36566G. P.V. and M.V. were supported by CSF Grant No. 15-000262S and M.V. also by Grant SGS16/ 249/OHK4/3T/14. V.K. was supported by Grant SGS16/245/OHK4/ $3 \mathrm{~T} / 14$. 


\section{References}

1. Coey JMD (2010) Magnetism and magnetic materials. Cambridge University Press, Cambridge

2. Heczko O, Scheerbaum N, Gutfleisch O (2009) Magnetic shape memory phenomena. In: Liu JP et al (eds) Nanoscale magnetic materials and applications. Springer, New York, pp 399-439

3. Acet M, Manosa L, Planes A (2011) Magnetic-field-induced effects in martensitic Heusler-based magnetic shape memory alloys. In: Buschow KHJ (ed) Handbook of magnetic materials, vol 19. Elsevier, Amsterdam, pp 231-290

4. Ullakko K, Huang JK, Kanter C, Kokorin VV, O'Handley RC (1996) Large magnetic-field-induced strains in $\mathrm{Ni}_{2} \mathrm{MnGa}$ single crystals. Appl Phys Lett 69:1966

5. Heczko O, Sozinov A, Ullakko K (2000) Giant field-induced reversible strain in magnetic shape memory NiMnGa alloy. IEEE Trans Magn 36:3266-3268

6. Likhachev AA, Ullakko K (2000) Magnetic-field-controlled twin boundaries motion and giant magneto-mechanical effects in $\mathrm{Ni}-$ Mn-Ga shape memory alloy. Phys Lett A 275:142

7. Sozinov A, Lanska N, Soroka A, Zou W (2013) 12\% magnetic field-induced strain in Ni-Mn-Ga-based non-modulated martensite. Appl Phys Lett 102:021902

8. Kainuma R et al (2006) Magnetic-field-induced shape recovery by reverse phase transformation. Nature 439:957-960

9. Heczko O (2014) Magnetic shape memory effect and highly mobile twin boundaries. Mater Sci Technol 30:1559-1578

10. L'vov V, Zagorodnyuk S, Chernenko V, Takagi T (2002) Magnetic-field-induced stresses and magnetostrain effect in martensite. Mater Trans JIM 43:876-880

11. Chernenko VA, L'vov VA (2008) Magnetoelastic nature of ferromagnetic shape memory effect. Mater Sci Forum 583:1-20

12. L'vov VA, Chernenko VA (2011) Magnetic anisotropy of ferromagnetic martensites. Mater Sci Forum 684:31-47

13. Mullner P, Chernenko VA, Kostorz G (2003) A microscopic approach to the magnetic-field-induced deformation of martensite (magnetoplasticity). J Magn Magn Mater 267:325-334

14. Chmielus M, Witherspoon C, Ullakko K, Müllner P, Schneider R (2011) "Effects of surface damage on twinning stress and the stability of twin microstructures of magnetic shape memory alloys. Acta Mater 59:2948-2956

15. Thomas M, Heczko O, Buschbeck J, Lai YW, McCord J, Schultz L, Fahler S (2009) Stray-field-induced actuation of free-standing magnetic shape-memory films. Adv Mater 21:3708

16. Aaltio I et al (2008) Temperature dependence of the damping properties of Ni-Mn-Ga alloys. Mater Sci Eng A 481:314-317

17. Srivastava V, Song Y, Bhatti K, James RD (2011) The direct conversion of heat to electricity using multiferroic alloys. Adv Energy Mater 1:97-104

18. Straka L, Soroka A, Heczko O, Hanninen H, Sozinov A (2014) Mechanically induced demagnetization and remanent magnetization rotation in $\mathrm{Ni}-\mathrm{Mn}-\mathrm{Ga}(-\mathrm{B})$ magnetic shape memory alloy. Scr Mater 87:25

19. Heczko O, Drahokoupil J, Straka L (2015) Enhanced magnetic hysteresis in Ni-Mn-Ga single crystal and its influence on magnetic shape memory effect. J Appl Phys 117:17E703

20. Gaitzsch U, Pötschke M, Roth S, Rellinghaus B, Schultz L (2009) A $1 \%$ magnetostrain in polycrystalline $5 \mathrm{M} \mathrm{Ni-Mn-Ga.} \mathrm{Acta}$ Mater 57:365-370

21. Straka L, Heczko O, Hänninen H (2008) Activation of magnetic shape memory effect in Ni-Mn-Ga alloys by mechanical and magnetic treatment. Acta Mater 56:5492-5499

22. Chmielus M, Chernenko VA, Knowlton WB, Kostorz G, Mullner $P$ (2008) Training, constraints, and high-cycle magneto- mechanical properties of Ni-Mn-Ga magnetic shape-memory alloys. Eur Phys J Spec Top 158:79-85

23. Heczko O, Straka L, Soderberg O, Hannula SP (2005) Magnetic shape memory fatigue. Smart structures and materials, active materials behavior and mechanics. Book series proc. SPIE 5761(2005):513-520

24. Aaltio I et al (2010) High-cycle fatigue of $10 \mathrm{M} \mathrm{Ni-Mn-Ga}$ magnetic shape memory alloy in reversed mechanical loading. Smart Mater Struc 19:075014

25. Straka $L$ et al (2016) The relation between lattice parameters and very low twinning stress in $\mathrm{Ni}_{50} \mathrm{Mn}_{25+\mathrm{x}} \mathrm{Ga}_{25-\mathrm{x}}$ magnetic shape memory alloys. Smart Mater Struct 25:025001

26. Straka L et al (2003) Study of austenite-martensite transformation in Ni-Mn-Ga magnetic shape memory alloy. J de Phys IV 112:911-915

27. Pinneker V, Gueltig M, Sozinov A, Kohl M (2014) Single phase boundary actuation of a ferromagnetic shape memory foil. Acta Mater 64:179-187

28. Krenke T, Duman E, Acet M, Wasserman EF, Moya X, Manosa L, Planes A (2005) Inverse magnetocaloric effect in ferromagnetic Ni-Mn-Sn alloys. Nat Mater 4:450

29. Niemann $R$ et al (2010) Metamagnetic transitions and magnetocaloric effect in epitaxial Ni-Co-Mn-In films. Appl Phys Lett 97:222507

30. Tegus O, Bruck E, Buschow KHJ, de Boer FR (2002) Transitionmetal-based magnetic refrigerants for room-temperature applications. Nature 415:150

31. Heczko O, Straka L, Seiner H (2013) Different microstructures of mobile twin boundaries in $10 \mathrm{M}$ modulated Ni-Mn-Ga martensite. Acta Mater 61:622-631

32. Straka L et al (2011) Twin interaction and large magnetoelasticity in Ni-Mn-Ga single crystals. J Appl Phys 109:063504

33. Straka L, Heczko O (2003) Superelastic response of Ni-Mn-Ga martensite in magnetic fields and a simple model. IEEE Trans Magn 39:3402-3404

34. Bhattacharya K (2003) Microstructure of martensite. Oxford University Press, New York

35. Straka L et al (2011) Highly mobile twinned interface in $10 \mathrm{M}$ modulated Ni-Mn-Ga martensite: Analysis beyond the tetragonal approximation of lattice. Acta Mater 59:7450-7462

36. Seiner H, Straka L, Heczko O (2014) A microstructural model of motion of macro-twin interfaces in Ni-Mn-Ga $10 \mathrm{M}$ martensite. J Mech Phys Solids 64:198-211

37. Straka L, Hanninen H, Heczko O (2011) Temperature dependence of single twin boundary motion in Ni-Mn-Ga martensite. Appl Phys Lett 98:141902

38. Heczko O, Soroka A, Hannula SP (2008) Magnetic shape memory effect in thin foils. Appl Phys Lett 93:022503

39. Molnar P et al (2008) In situ neutron diffraction study of magnetic field induced martensite reorientation in $\mathrm{Ni}-\mathrm{Mn}-\mathrm{Ga}$ under constant stress. J Phys 20:104224

40. Heczko O (2014) Understanding motion of twin boundary-a key to magnetic shape memory effect. IEEE Trans Magn 50:2505807

41. Kopecký V, Perevertov O, Straka L, Ševčík M, Heczko O (2015) Equivalence of mechanical and magnetic force in magnetic shape memory effect. Acta Phys Pol A 128:754-757

42. Novák V, Šittner P, Ignácová S, Černoch T (2006) Transformation behaviour of prism-shaped shape memory alloy single crystals. Mater Sci Eng A 438-440:755-762

43. Seiner $H$ (2015) Mobile interfacial microstructures in single crystals of $\mathrm{Cu}-\mathrm{Al}-\mathrm{Ni}$, shape memory alloy. Shap Mem Superelast $1: 268-274$

44. Zelený $\mathrm{M}$ et al (2014) First-principles study of Co- and Cu-doped $\mathrm{Ni}_{2} \mathrm{MnGa}$ along the tetragonal deformation path. Phys Rev B 89:184103 
45. Straka L (2013) Effect of intermartensite transformation on twinning stress in Ni-Mn-Ga 10M martensite. J Appl Phys 114:063504

46. Kucza N et al (2015) Magnetic-field-induced bending and straining of Ni-Mn-Ga single crystal beams with high aspect ratios. Acta Mater 95:284-290

47. Ullakko K, Wendell L, Smith A, Müllner P, Hampikian G (2012) A magnetic shape memory micropump: contact-free, and compatible with PCR and human DNA profiling. Smart Mater Struct 21:115020

48. Thomas M et al (2008) Magnetically induced reorientation of martensite variants in constrained epitaxial $\mathrm{Ni}-\mathrm{Mn}-\mathrm{Ga}$ films grown on $\mathrm{MgO}(001)$. New J Phys 10:023040

49. Chluba C, Ge W, Miranda RL, Strobel J, Kienle L, Quandt E, Wuttig M (2015) Ultralow-fatigue shape memory alloy films. Science 348:1004-1007 